\title{
Kernos
}

Revue internationale et pluridisciplinaire de religion grecque antique

1 | 1988

Varia

\section{Les origines de la déesse Athéna. Un réexamen de l'évidence}

\section{Nicolaos Papachatzis}

\section{OpenEdition \\ Journals}

\section{Édition électronique}

URL : http://journals.openedition.org/kernos/85

DOI : $10.4000 /$ kernos.85

ISSN : 2034-7871

\section{Éditeur}

Centre international d'étude de la religion grecque antique

\section{Édition imprimée}

Date de publication : 1 janvier 1988

ISSN : 0776-3824

\section{Référence électronique}

Nicolaos Papachatzis, "Les origines de la déesse Athéna. Un réexamen de l'évidence », Kernos [En ligne], 1 | 1988, mis en ligne le 31 janvier 2011, consulté le 01 mai 2019. URL : http:// journals.openedition.org/kernos/85; DOI : 10.4000/kernos.85 


\title{
L'ORIGINE DE LA DÉESSE ATHÉNA : UN RÉEXAMEN DE L'ÉVIDENCE
}

\begin{abstract}
Athéna, tout comme d'autres divinités des temps historiques, avait hérité de caractères non seulement de divinités minoennes mais aussi de dieux ou démons traditionnels de Grèce continentale.

Martin Pers. Nilsson, influencé par le charme de la civilisation crétoise préhistorique que le monde découvrait alors en ce premier quart du siècle, a surestimé les traits minoens d'Athéna et soutenu son origine minoenne. La première étude qu'il publia sur le sujet parut à Copenhague, en 1921, sous le titre Die Anfänge der Göttin Athena (Det Kgl. Danske Videnskabernes Selskab, Hist.-Philol. Medd. IV, 7). Nilsson exprima à nouveau son point de vue dans une courte histoire de la religion grecque publiée en Grande Bretagne, en 1925, et intitulée A History of Greek Religion (ainsi que dans une nouvelle édition du même livre parue en 1949) et le reprit sans modification dans le premier volume de sa Geschichte der griechischen Religion (5 Abt., 2 des Handbuch der Altertumswissenschaft), 1941 (ainsi que dans la troisième édition de 1967), comme dans la monographie étendue The Minoan-Mycenaean Religion parue en 1927, aux pages 415 et suivantes, et plus spécialement dans la dernière édition de ce livre (1950), aux pages 485 et suivantes.
\end{abstract}

\section{Le point de vue de Nilsson}

Dans ces travaux (ainsi que dans quelques autres de moindre importance que Nilsson publiait sporadiquement), Athéna est constamment qualifiée de «direct descendant of minoan palace goddess» (p. 498 de la monographie citée en dernier lieu, dans l'édition de 1950 à laquelle se rapportent également les renvois ci-dessous). Pour cette déesse crétoise il n'existait pas de statues monumentales, mais uniquement des figurines qui la représentaient accompagnée d'un seul ou de plusieurs serpents auxquels elle doit sa dénomination de «déesse aux serpents» (snake goddess). Un oiseau accompagne également la représentation de la déesse. Le desservant du 


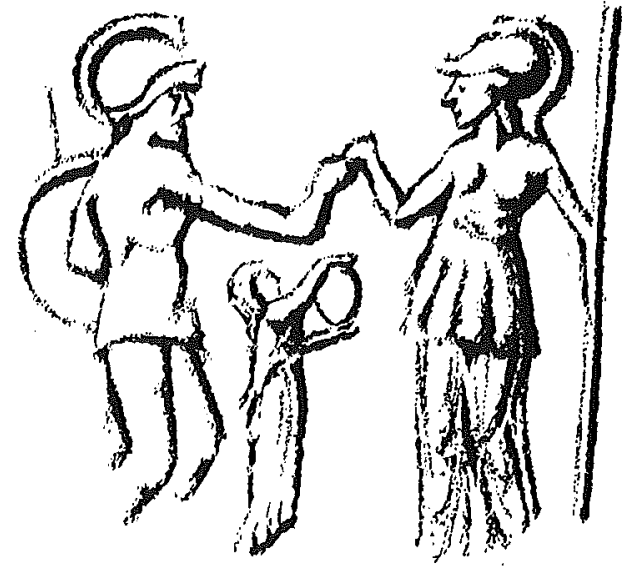

Fig. 3. Kepheus, Athéna et Stéropè representés sur une monnaie provenant de Tégée.

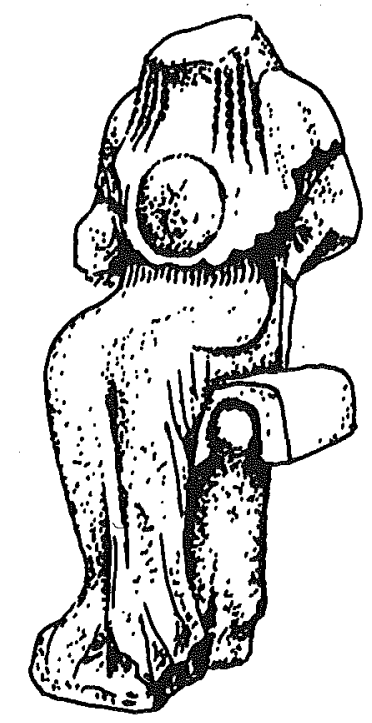

Fig. 4. Statue en pierre d'Athéna trônant attribuée à Endoios

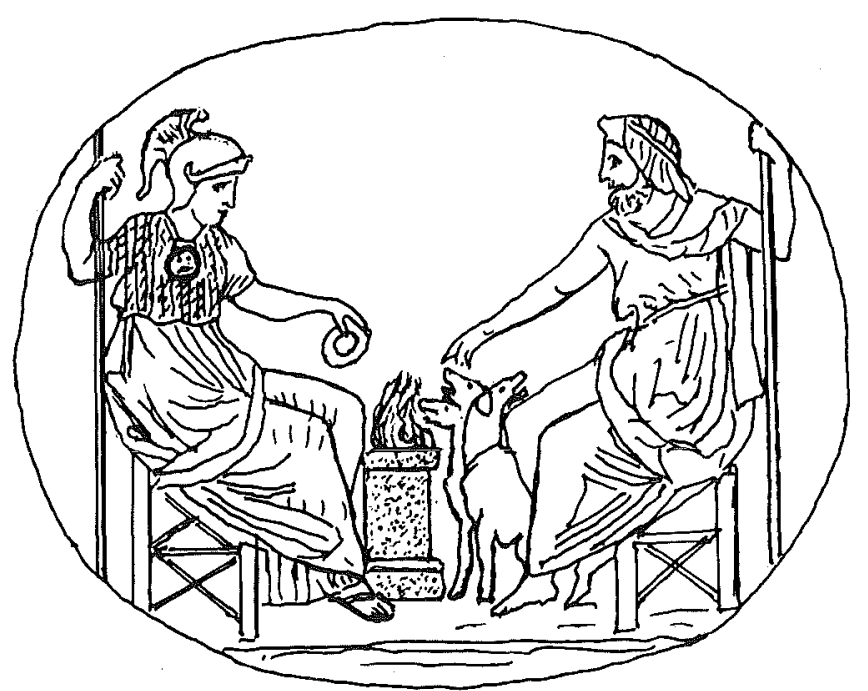

Fig. 5. Gemme florentine représentant Athéna, Hadès et Cerbère. 
quantité considérable d'objets minoens découverts à Mycènes est précisément absente «la déesse aux serpents», que beaucoup aujourd'hui considèrent comme exclusivement crétoise ${ }^{1}$.

Un lieu cultuel semblable à celui du palais crétois de Cnossos n'a pas été découvert au mégaron de Mycènes, ni même dans la cour, bien que la fouille ait atteint le rocher naturel ${ }^{2}$. Le temple archaïque construit au sommet de la colline et celui qui l'a remplacé aux temps hellénistiques se trouvaient à l'extérieur des ruines du mégaron mycénien ${ }^{3}$. Le roi mycénien n'était pas chargé des obligations sacerdotales (comme le soutenait Nilsson), parce qu'à Mycènes existait un collège de prêtres autonome, indépendant du pouvoir du chef politique comme il apparaît dans les tablettes du XIIIe s. av. J.C. A cette époque, à Mycènes, le culte public était pratiqué dans un lieu particulier, à l'extrémité sud-occidentale de l'acropole, accessible du palais ainsi que de la porte aux lionnes par une sorte de «voie sacrée». Cet endroit, dont une partie avait fait anciennement l'objet d'une fouille de Chr. Tsountas et de A. Wace, a été fouillé dans son ensemble par G. Mylonas et W. Taylour. Il y avait là un «temple» avec un autel circulaire en maçonnerie, d'un diamètre de $1,40 \mathrm{~m}$, à l'est. On a retrouvé également des vestiges d'un autre autel plus ancien. Une seconde partie du lieu cultuel fut dégagée à peu de distance vers l'ouest et à un niveau inférieur : elle était destinée aux dieux et aux démons du monde infernal, pour lesquels était prévu une forme de culte différente, consistant en sacrifices expiatoires ('́v $\alpha \gamma \downarrow \sigma \mu o \hat{)}$ ) et en exorcismes. Des idoles en terre cuite d'hideuses créatures du monde infernal y étaient conservées, ainsi que de nombreux simulacres de serpent. Cette découverte ne laisse aucun doute sur la vigueur extraordinaire que connaissaient les cultes chthoniens populaires à Mycènes au XIIIe s. av. J.-C. et accentue d'autant le gouffre séparant la vie religieuse de la Crète minoenne de celle de la Grèce mycénienne ${ }^{4}$.

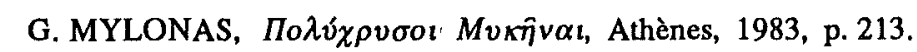

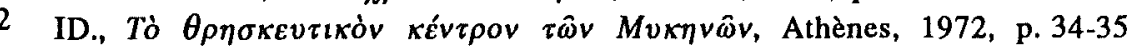
(publication de l'Académie d'Athènes).

3 Loc.cit.

4 Les premiers rapports de fouille de W. TAYLOUR concernant le sanctuaire des démons sont Mycenae. 1968, in Antiquity (1969), p. 91-97, et New Light on Mycenaean Religion, in Antiquity (1970), p. 270-280. 
Ce double lieu sacré reçut le nom de «centre cultuel de Mycènes» et son indépendance du palais a été vivement soulignée. On a soutenu l'existence à Pylos d'un semblable lieu, indépendant du palais, sur base des tablettes inscrites. On a également découvert à Pylos un petit sèkos, non loin du palais, dont l'entrée était précédée d'un autel en pierre (à l'instar des autels des temples des temps historiques). De semblables sèkoi ont aussi été trouvés dans l'acropole basse de Tirynthe, à proximité d'habitations ${ }^{5}$.

b. Le serpent qui accompagne l'image de la déesse minoenne des palais et celle d'Athéna constitue simplement un indice de la nature chthonienne de ces deux déesses. Des divinités associées au monde infernal ou issues de celui-ci avaient comme attribut principal le serpent. Parmi ces divinités figurent, par exemple, Zeus Meilichios, Zeus Ktèsios, Asklépios et la multitude de dieux ou héros guérisseurs topiques, ainsi qu'Hermès psychopompe. La fouille de Taylour a montré que le serpent (représenté d'une façon si particulière que toute influence crétoise est exclue) était même aux temps préhistoriques plus courant en Grèce continentale qu'en Crète.

Nilsson n'a pas accepté le point de vue d'Evans selon lequel la «déesse aux serpents» crétoise était une créature du monde infernal, «parce qu'elle était vénérée dans les habitations». Il a refusé par conséquent la nature chthonienne du serpent qui l'accompagne, et le rangea dans la classe des serpents bienveillants, celle de l'oikovpòs ö $\varphi$ is. Toutefois, la distinction des serpents de nature bienveillante et de nature malveillante annule la raison du culte qui vise justement à convertir la force ennemie en une force amie et, par conséquent, à faire du seul et unique serpent qui intéresse la croyance religieuse une puissance amie pour celui qui le vénère et hostile envers tous les autres. S'il existait un serpent de nature inoffensive et bienveillante, il n'aurait été l'objet d'un culte de la part de personne. Malfaisant, l'était également le serpent figuré en Crète habituellement sur un type de petites amulettes qui ont la forme de petites pommes de pin. Le serpent devenait protecteur de celui qui portait l'amulette et ennemi de ceux qui lui voulaient du mal. De la même manière

5 K. KILIAN, in $A A$ (1979), p. 390. Cf. aussi $A A$ (1978), (1981), (1982), (1983). 
l'oikovpòs ö $\varphi ı \varsigma$ protégeait la demeure et ses habitants qui lui offraient la $\mu \varepsilon \lambda \iota \tau o ́ \varepsilon \sigma \sigma \alpha$ et était hostile à tous les autres.

c. Un oiseau accompagne également les images des déesses minoenne et grecque. L'oiseau de la première est d'une espèce indéterminée, celui d'Athéna est une chouette. Elle a été associée à Athéna, selon Nilsson, uniquement pour la raison que beaucoup de chouettes ont leurs nids dans les failles du rocher de l'acropole athénienne (p. 496).

Dans les traditions populaires, la chouette devait être attachée à Athéna bien avant qu'elle n'apparaisse dans les représentations figurées de la déesse, qui ne sont pas antérieures au début des temps historiques. L'association iconographique de la chouette et d'Athéna avait rendu encore plus évidents les liens étroits d'Athéna avec le monde infernal, en raison de la croyance populaire bien connue en Grèce (et ailleurs), qui associe la chouette et la mort ${ }^{6}$. Athéna était une créature du monde infernal avant de prendre place dans le panthéon olympien. Nilsson ne croit pas que la chouette pouvait avoir été attachée à Athéna avant les temps historiques, parce qu'«il ne connaît pas de représentations de chouettes sur les monuments minoens et mycéniens» (p. 494). Bien auparavant, cependant, $\mathrm{K}$. Müller, dans sa publication des objets découverts par W. Dörpfeld provenant des trois tombes mycéniennes à tholos de Kakovatos?, mentionne une chouette figurée au repos sur une lame d'or, percée aux deux extrémités de deux trous (peut-être parce qu'elle était clouée sur un cercueil en bois ou sur tout autre objet en bois ou en or). Müller lui-même compare cette chouette avec les chouettes crétoises que comprend le rapport de fouille d'Evans de l'année $1903^{8}$. Sp. Marinatos fait mention de deux chouettes en or (l'une au repos, comme celle de Kakovatos, et l'autre en vol) provenant de sa fouille d'une tombe mycénienne à Peristeria en Messénie. Il signale également des chouettes trouvées par C. Blegen dans une tombe à tholos d'Eglianos, à proximité du palais mycénien ${ }^{9}$. Marinatos ${ }^{10}$

6 Cf. Sp. MARINATOS, Die Eulengöttin von Pylos, in AM, 83(1968), p. 167 sq.

$7 A M, 34(1909)$, p. 269 sq.

8 BSA, 9(1902-1903), p. 56.

$9 A J A, 58(1954)$, p. 22.

10 Die Eulengöttin von Pylos, in $A M, 83(1968)$, p. 107 sq. 
croyait que la chouette «était présente» dans chaque tombe mycénienne de Messénie, et cette opinion sous-tend son étude sur l'existence à Pylos d'une déesse de la mort qui avait pour attribut la. chouette.

d. Quant aux deux passages homériques où Nilsson trouve un souvenir d'un culte préhistorique d'Athéna à l'intérieur du palais mycénien, il suffit de rappeler l'interprétation plus logique qui en a été proposée, et connue depuis longtemps ${ }^{11}$. Le premier passage extrait du Catalogue des Vaisseaux de l'Iliade (II, 546-599) appartient à l'ensemble des onze vers (546-556) considérés depuis l'antiquité comme une interpolation de l'époque de Pisistrate ${ }^{12}$. L'addition de ces vers visait à mettre en valeur la contribution de l'Athènes mycénienne à la guerre de Troie. Indépendamment de cette intention chauvine qu'avait l'interpolation, l'auteur de ces onze vers connaissait la légende locale de la naissance d'Erechthée ou Erichthonios et savait aussi qu'Athéna reconnut pour sien le

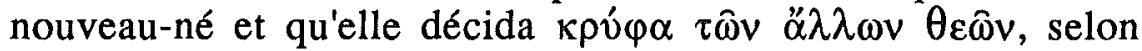
l'expression d'Apollodore (III, 14, 6), de l'élever elle-même dans son temenos. Elle enferma l'enfant dans une ciste avec un serpent pour garde et cacha la ciste, selon le vers 549 du chant II de l'Iliade,

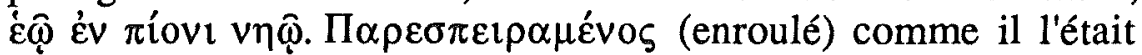
dans la ciste, le serpent, accomplissant son devoir, punit sévèrement les Cécropides qui l'ouvrirent, et Athéna acheva l'éducation de l'enfant dans son temple (comme le rapporte le vers 548 de l'Iliade). Une fois Erechthée ou Erichthonios devenu grand, il devint roi d'Athènes et c'est lui le premier qui dressa un xoanon d'Athéna dans le temple. A sa mort, Erechthée fut enseveli dans le sanctuaire même d'Athéna.

Le second passage homérique (Od., VII, 78-81) suppose connue l'existence du dit ancien temple d'Erechthée (ou pré-Erechtheion)

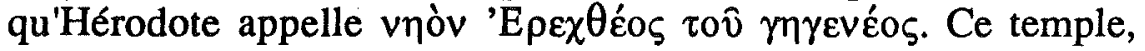
tout comme l'Erechtheion des temps classiques, était «double»; il

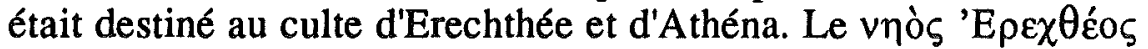

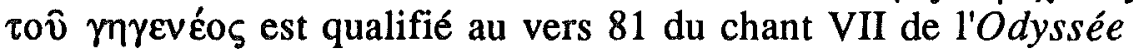

11 Cf. Aug. FRICKENHAUS, Das Athenabild des alten Tempels in Athen, in AM

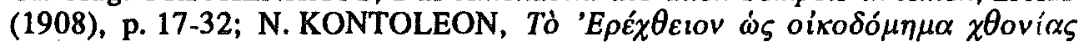
$\lambda \alpha \tau \rho \varepsilon i \alpha \varsigma$, Athènes, 1949, p. 3 sq.

12 DIOG. LAERT, I, 57. 


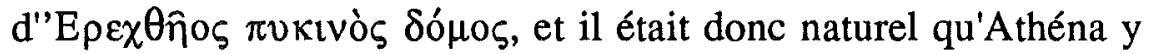
pénètre dès son arrivée, puisque l'acropole était encore sans les temples prestigieux construits exclusivement pour elle.

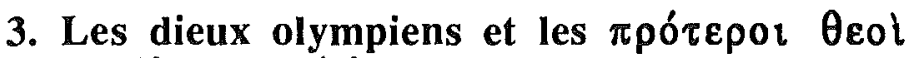 ou dieux antérieurs}

Pour les Grecs, spécialement pour les plus anciens, sans qu'ils aient eu connaissance de la religion de la Crète minoenne, les dieux de l'Olympe n'étaient pas anciens. Eschyle les dit nouveaux ${ }^{13}$ ou jeunes ${ }^{14}$ dieux, parce qu'avant eux d'autres dieux ou démons, sauvages et inhumains, dominaient le monde. La Théogonie d'Hésiode qualifie ces dieux sauvages de $\pi \rho{ }^{\prime} \tau \varepsilon \rho \mathrm{\imath}^{15}$ et Eschyle,

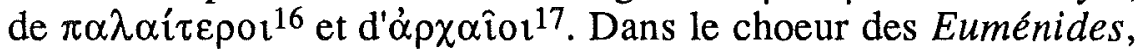
ce dernier présente les anciennes déesses Erinyes avec toute la sauvagerie que leur reconnaissaient les traditions populaires de l'époque, pour justifier leur qualification de $\mu \iota \sigma \eta \dot{\eta} \mu \alpha \alpha \dot{\alpha} v \delta \rho \hat{\omega} v$ $\kappa \alpha i \quad \theta \varepsilon \hat{\omega} \vee$ ò $\lambda v \mu \pi i \omega^{18}$. Les Erinyes, dans l'Iliade, sont des divinités $\sigma \tau v \gamma \varepsilon \rho \alpha i^{19}$.

Il y avait cependant des créatures infernales plus sauvages que les Erinyes et plus obscures, qui faisaient de la religion pré-olympienne une véritable «religion de terreur» ${ }^{20}$. Le sanctuaire du versant sud-occidental de l'acropole de Mycènes qu'a fouillé $\mathrm{W}$. Taylour en 1968 et 1969 a livré des indices de créatures de cette nature. Une vingtaine d'idoles de démons ou de dieux d'aspect repoussant ou même terrifiant a été découverte dans ce sanctuaire de dimensions relativement réduites (fig. 1). Plus de quinze simulacres de serpent de grande taille ont indiqué d'une manière évidente la nature infernale du sanctuaire. Les idoles anthropomorphes étaient en majorité féminines et leur coloration en intensifiait les caractères terrifiants ou

13 ESCH., Prom., 439.

14 ESCH., Eum., 778 sq., 808 sq.

15 HES., Theog., 424.

16 ESCH., Eum., 721.

17 Ibid., 728.

18 lbid., 73.

19 Il, LX, 454.

20 «Religion of Terror», ce sont les termes de J.E. HARRISON (Proleg., 1903, p. 188; Epileg., 1921, p. 4). 
repoussants. Il en va de même de la coloration des serpents qui étaient en terre cuite, tout comme les idoles de dieux ou démons ${ }^{21}$. A l'époque historique il y avait à Corinthe le $\mu v \hat{\eta} \mu \alpha$ des jeunes démons Pheres et Mermeros (le Fort et le Terrible), qui provoquaient la mort des jeunes enfants lorsque les Corinthiens négligeaient de célébrer les rites expiatoires adéquats. Le mnèma était un sanctuaire de culte chthonien, gardé par une terrible créature féminine du monde infernal, qui a subsisté jusqu'à l'époque de Pausanias (alors que n'étaient plus pratiqués les rites d'apaisement pour les jeunes démons). La gardienne du mnema était عíóv

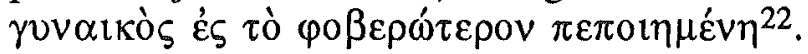

\section{Gorgô ou Méduse et Athéna}

De toutes les figures pré-olympiennes du monde infernal, la plus importante était Gorgô ou Méduse. Les traditions en colportent l'image d'une créature destructrice contre laquelle n'existait aucun moyen de se défendre, et dont personne ne pouvait modifier les dispositions par une quelconque sorte de culte ou par des rites «énagistiques» ou des exorcismes. Dans l'Iliade, Gorgô est

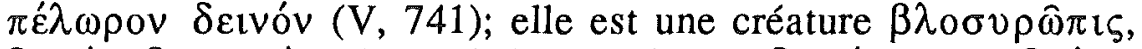

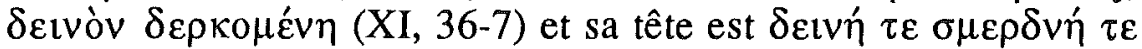
$(\mathrm{V}, 742)$. Pour les peintres et les sculpteurs de l'époque archaïque, elle est une créature étrange : des ailes sortent de ses épaules, des serpents se mêlent aux tresses de sa chevelure tandis que d'autres lui ceignent la taille (fig. 2).

21 A l'époque mycénienne, les cultes populaires n'étaient pas dédaignés, mais ils étaient célébrés parallèlement au culte officiel lorsque l'exigeaient les circonstances. C'est la raison pour laquelle le sanctuaire des démons infernaux était si proche du lieu des cérémonies cultuelles officielles. En règle générale, les épidémies, de même que la destruction des récoltes, étaient attribuées à la colère des démons du monde infernal, qu'il était alors nécessaire de calmer par des rites d'apaisement. Aux temps historiques, la plupart des sanctuaires des divinités du monde infernal demeuraient souvent fermés et ne s'ouvraient qu'un

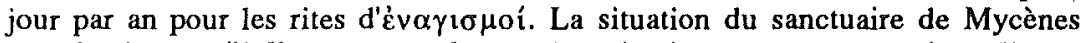
se prête à ce qu'il fût ouvert seulement lors de circonstances exceptionnelles.

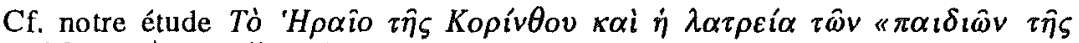

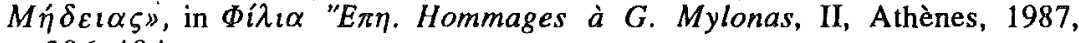
p. 396-404. 
Les traditions présentent la Gorgone Méduse établie avec deux autres Gorgones sur la route qui mène au monde infernal ${ }^{23}$. Sur cette même voie étaient placées deux autres soeurs des Gorgones, les Graiai ${ }^{24}$. Comme celles-ci, les Gorgones ont dû être originellement deux en nombre; par la suite leur fut jointe une troisième, Méduse (la Souveraine), au passé plus illustre, comme en témoigne son nom. C'est la raison pour laquelle Méduse s'est rapidement distinguée de ses deux soeurs et qu'elle est devenue la Gorgone par excellence. Peut-être était-elle une reine de l'au-delà propre à une certaine contrée, qui s'était trouvée, grâce à cette haute qualification, parèdre du souverain infernal Poséidon et mère des deux enfants de celui-ci. Abaissée au rang de Gorgone et privée de l'immortalité, elle en a néanmoins conservé l'arme de la reine souterraine, à savoir le pouvoir de tuer par son regard.

Selon la légende, Polydektes, autre roi souterrain (que son nom désigne simplement comme une forme d'Hadès, «l'hôte d'une grande foule»), a entrepris, par l'intermédiaire de Persée, de l'éliminer dans le dessein de s'approprier sa force et d'échapper aux limites étroites de l'île de Sériphos. Cependant, la seule à avoir bénéficié de la décapitation de Méduse, c'est Athéna, déesse chthonienne elle aussi et directement intéressée par le pouvoir de la Gorgone. Selon les légendes attiques, c'est Athéna elle-même qui a

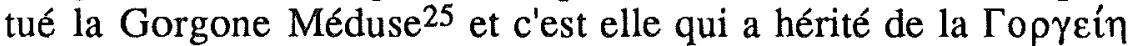
$\kappa \varepsilon \varphi \alpha \lambda \grave{n}$ qui l'a rendue invincible et qui fut la raison de sa mutation en déesse guerrière (Athéna avait triomphé des Géants à l'aide de cette arme avant que les peintres et les sculpteurs ne la représentent les combattant avec des armes conventionnelles). Persée, dont d'autres légendes font le meurtrier de Méduse, avait réussi son oeu-

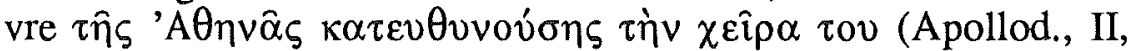
$4,2)$; il est donc justifié que certains aient prétendu que toute cette

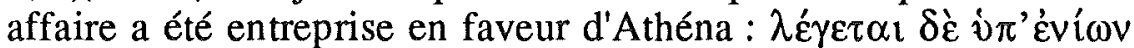

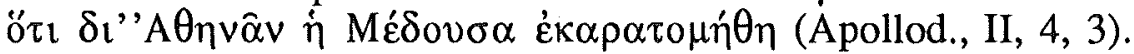
En réalité, Athéna a absorbé la Gorgone Méduse ou bien elle l'a

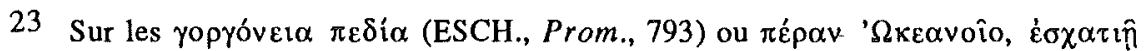
$\pi$ ò̀ v vikìs (HES., Theog., 274-275).

24 Cf. HES., Theog., 273.

25 EUR., Ion, 989-991. 
soumise à sa propre essence. C'est pourquoi incontestablement la déesse s'identifiait souvent à elle ${ }^{26}$.

Ni la recherche du prototype de la Gorgone Méduse (était-il oriental, grec ou crétois ?), ni la question de l'antériorité du gorgoneion à la Gorgone Méduse ou vice versa, ni encore celle de l'existence d'une quelconque forme de culte ou de rites «énagistiques» pour cette créature monstrueuse ne peut éclairer la relation entre Athéna et Méduse. Il importe seulement que l'imagination populaire ait conçu Méduse détentrice d'un pouvoir, celui de pétrifier au moyen du regard. Si à l'origine seul le gor-

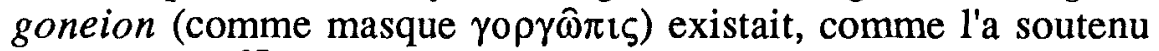
J.E. Harrison 27, ce pouvoir lui était alors attribué, mais il était plus intense que celui des masques rituels, avec lesquels Harrison compare le gorgoneion. Le masque de la Kidaria des Phénéates, par exemple, était porté par le prêtre lors d'un rite mystique pour qu'il puisse, dissimulé derrière lui, battre sans crainte les puissances souterraines malveillantes : le masque hideux provoque la peur; les sombres puissances souterraines sont terrifiées pendant que le prêtre frappe le sol avec des baguettes et elles prennent la fuite, libérant la ville et la contrée environnante de leur action destructive ${ }^{28}$. Les Anciens attribuaient au gorgoneion une action exterminatrice envers tout être vivant auquel il ferait face. A cet égard, la tradition tégéate à propos d'un cadeau d'Athéna qui contribua à ce que Tégée la reconnût déesse poliade est instructive : Athéna était apparue au roi de Tégée Kepheus et avait offert à la fille de ce dernier, Stéropè, une boucle de la chevelure de la Gorgone Méduse dans une hydrie de

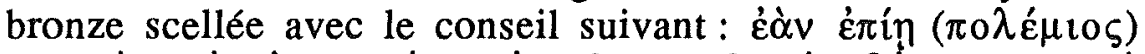

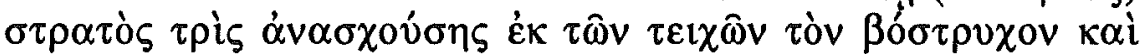

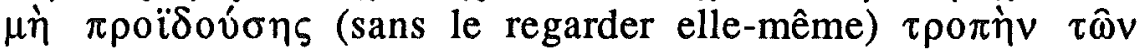

26 Dans un fragment conservé de cet auteur curieux des antiquités, vivant à l'époque hellénistique, qu'était Palaephatos, il est rapporté que les habitants de lîle de Kemè (a l'ouest de la Libye) appelaient Athéna Gorgô. Aux temps modemes, O. GRUPPE (Mythol. u. Religionsgesch., 2, 1193-1201), examinant le pouvoir de pétrification qu'a la tête de Gorgô, la considère comme tête d'Athéna: «aber ohne Frage ursprünglich ihr eigenes Haupt ist». - Dans I'Odyssée (XI, 633), la tête de Gorgô est décrite comme arme de la reine des Enfers Perséphone, qui pouvait s'en servir pour mettre un terme à la vie des mortels indésirables. Cette arme est donc passée en la possession d'une déesse à l'origine apparentée.

.

28 PAUS., VIII, 15, 1 sq. 


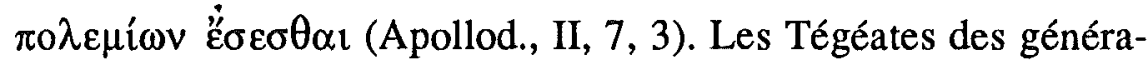
tions suivantes ont représenté sur leur monnaie le roi Kepheus en compagnie d'Athéna et, au milieu d'eux, Stéropè munie de l'hydrie contenant la funeste mèche de cheveux, et veillant à ne pas la regarder (fig. 3). Pausanias ajoute que les Tégéates, dont le temple le plus officiel était consacré à Alea, ont fondé un autre sanctuaire pour Athéna $\pi \circ \lambda \iota \hat{\alpha} \tau \iota \varsigma$ qu'ils appelaient "E $\rho v \mu \alpha$ (sanctuaire de défense ou de protection) et ont enjoint que le prêtre y pénètre une fois l'an lors d'une cérémonie particulière (VIII, 47, 5).

\section{Athéna dans les arts figuratifs}

Les peintres et les sculpteurs, depuis le Géométrique récent mais surtout à partir de l'époque archaïque, avaient commencé à figurer Athéna d'abord en vénérable souveraine de l'au-delà, et ensuite en déesse guerrière. Dans le premier type, la déesse était assise sur un trône; elle était coiffée du polos, munie de l'égide et du gorgoneion sur la poitrine, mais dépourvue d'armes. Le second type la montre debout et tout armée. Il est évident que les artistes ont eu recours aux armes conventionnelles (lance, bouclier et casque) une fois que s'est perdue dans l'oubli la puissance destructrice du gorgoneion. Chez Homère, l'activité d'Athéna est militaire. Il est cependant significatif que le poète ne connaisse pas de statues cultuelles ni d'autres images de la déesse au combat ou tout au moins en armes. La seule image qu'il mentionne - et c'est la plus ancienne mention d'une image d'Athéna dans les témoignages littéraires - est la statue cultuelle de la déesse dans le temple de l'acropole d'Ilion, qui la représentait assise sur un trône. La reine Hécube, accompagnée d'autres femmes de Troie, lui apporta en offrande son peplos le plus précieux et le déposa à ses genoux (Il., VI, 303). Il est évident que l'auteur de ces vers connaissait une Athéna trônant telle que la figurent de nombreuses figurines archaïques, et cela malgré que la société chevaleresque de cette époque voulût la déesse triomphant de toute armée sur les champs de bataille. Et le plus ancien xoanon de la déesse qu'abritait son ancien temple sur l'acropole d'Athènes devait, lui aussi, la représenter assise sur un trône. Aux dires des

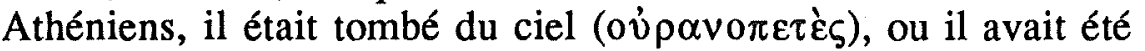
fabriqué en bois d'olivier par les soins d'Erichthonios ou de

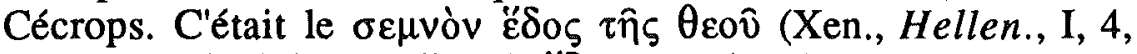

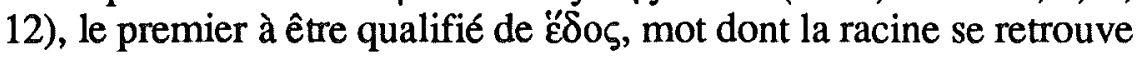




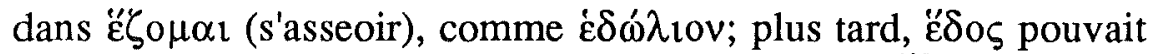
être toute statue cultuelle vénérable. C'est l'ancien "̌́oৎ d'Athéna qu'a dû reproduire le sculpteur Endoios qui travaillait à Athènes au temps de Pisistrate ou d'Hippias. Pausanias rapporte qu'à son époque encore la statue en pierre de l'Athéna assise d'Endoios était conservée à l'acropole d'Athènes. Certains en reconnaissent le type dans une statue trouvée au pied de l'acropole dans un état fragmentaire (sans tête ni bras), mais dont la poitrine s'orne de l'égide et du gorgoneion (fig. 4).

L'activité guerrière d'Athéna, telle que l'épopée l'a mise en valeur, a commencé à exercer une forte influence sur les peintres et les sculpteurs à partir de l'époque de Pisistrate ${ }^{29}$. Jusqu'alors, même lorsque l'esprit chevaleresque avait atteint son apogée, on était satisfait de l'image de la souveraine de l'au-delà trônant. Le culte d'Athéna au sanctuaire de Coronée en Béotie est un vif souvenir des anciennes relations de la déesse avec les divinités infer-

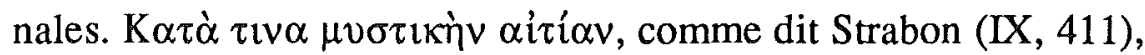
c'est-à-dire pour une raison révélée lors d'une cérémonie mystique dans le sanctuaire, l'image sculptée d'Athéna s'y dressait à côté de celle d'Hadès. Pausanias, qui a visité le sanctuaire, dit que la statue d'Athéna était élevée à côté de celle de Zeus en tant que roi souterrain de l'au-delà et qu'elles étaient toutes les deux des oeuvres d'Agoracrite (IX, 34, 1). Sur une gemme florentine, Athéna est représentée assise en face d'Hadès (ou, comme on l'a également soutenu, en face de Zeus chthonien ou encore de Sérapis, qui à l'époque romaine avait été identifié à Hadès). Entre ces deux figures, on peut voir Cerbère tricéphale et un autel (fig. 5).

\section{Athéna, déesse olympienne}

L'exemple de Tégée rapporté ci-dessus explique pourquoi les autres cités également veillaient à ce qu'Athéna soit vénérée sur leur acropole comme déesse poliade. Sur l'acropole de Lindos à Rhodes, on vénérait depuis une très haute antiquité une déesse locale de nature inconnue. A l'époque historique, les Doriens qui s'y installèrent ont consacré le promontoire rocheux et le sanctuaire de la

29 Aug. FRICKENHAUS, Das Athenabild des alten Tempels in Athen, in AM (1908), p. 25 : «Die Darstellung der Athena als Palladion beginnt in Athen mit und durch Peisistratos.» 
divinité qui y était honorée à «Athana». Comme l'a indiqué la fouille de son sanctuaire des temps historiques, cette Athana de Lindos ressemblait à l'Athéna de la Grèce continentale presque uniquement quant au nom ${ }^{30}$. Les deux autres cités de Rhodes (Ialysos et Camiros) firent elles aussi d'Athéna leur divinité poliade. Il en alla de même en Grèce continentale, où toutes les villes, influencées par l'épopée héroïque, s'étaient empressées, les unes après les autres, de fonder sur leur acropole des sanctuaires et des temples d'Athéna poliade, même si jusqu'alors elles honoraient comme protectrice une autre divinité locale. Pour l'Iliade (VI, 305),

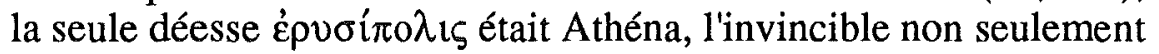
d'entre tous les mortels, mais encore d'entre tous les dieux. Les nobles, protecteurs des poètes épiques, étaient axés sur les joies de la vie et par conséquent hostiles aux divinités de sombre humeur de l'au-delà. Il était naturel que ceux-ci désirent que leur patronne fût Athéna qui, récemment et par les soins des nobles aristocrates, s'était introduite dans la famille des dieux ouraniens. Ce brusque passage d'Athéna s'est réalisé aux temps protogéométriques. Le symbole du nouveau «contenu» de la déesse est l'égide que les artistes ont représentée plus tard comme «peau de chèvre» sur sa poitrine, avec le gorgoneion. L'égide, $\alpha i \gamma i \zeta$, était en réalité une

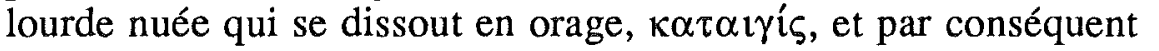
constituait un emblème approprié à une divinité ouranienne. C'était

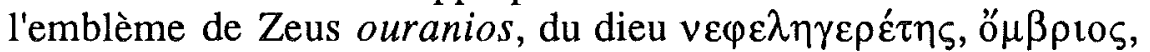

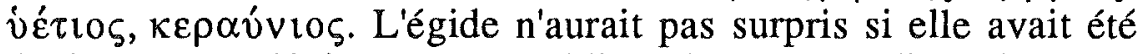
également conférée comme emblème à un autre dieu olympien (Apollon, par exemple), mais pas à un dieu chthonien.

C'est l'épopée héroïque qui a obligé Athéna à quitter sans tarder le panthéon chthonien qui était le sien jusqu'alors et de prendre une place au panthéon olympien. Le désir des nobles était encore que la nouvelle déesse olympienne occupe le plus vite possible la deuxième

30 Le connaisseur attentif de Lindos, Chr. BLINKENBERG, (La déesse de Lindos, in $A f R W, 28(1930)$, p. 154-165) la considérait comme une figure locale de

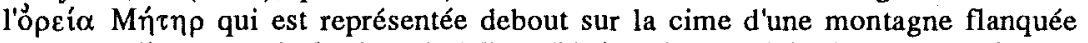
par deux lions, ou de la Grande Mère d'Asie mineure. Dès les plus anciennes figurines des temps historiques, elle apparaît comme protectrice du mariage et comme courotrophe. Dans les figurines de femmes porteuses de torches, BLINKENBERG (art. cit., p. 160) reconnaît les torches nuptiales du soir des noces. Il y a encore des statuettes de femmes serrant dans leurs bras un petit enfant et de nombreuses statuettes d'animaux, sauvages et domestiques, toutes offertes à Athéna Lindia. 
place, celle immédiatement après Zeus, dans le panthéon olympien. Les poètes qui voulaient dresser la généalogie des dieux s'en trouvèrent bien embarrassés. Finalement, ils ont présenté Athéna comme fille du dieu suprême, mais à la suite d'une naissance violente et surnaturelle.

Ainsi donc, on pourrait conclure qu'il est plus naturel de chercher l'ancêtre d'Athéna parmi les divinités pré-olympiennes de Grèce continentale, avec lesquelles s'était familiarisé l'élément helladique, surtout populaire, et non parmi les divinités minoennes qui étaient étrangères et difficilement compréhensibles. Avec le temps, le contenu d'Athéna s'est enrichi entre autres avec des traits de divinités minoennes qui n'étaient cependant pas les plus nombreux, ni les plus importants pour étayer le point de vue qu'il s'agit d'une déesse d'origine minoenne.

D. Voutsina 63, Cholargos,

Nicolaos PAPACHATZIS GR - 15561 ATHÈNES 